\title{
Horizons/Théâtre
}

Revue d'études théâtrales

$14 \mid 2019$

Les arts du spectacle dans l'Afrique subsaharienne - 2

\section{Le piège du folklore est totalement dépassé}

\section{Marjorie Bertin}

\section{(2) OpenEdition}

Journals

Édition électronique

URL : https://journals.openedition.org/ht/1760

DOI : $10.4000 /$ ht. 1760

ISSN : 2678-5420

\section{Éditeur}

Presses universitaires de Bordeaux

\section{Édition imprimée}

Date de publication : 1 janvier 2019

Pagination : 134-141

ISBN : 979-10-300-0718-3

ISSN : 2261-4591

\section{Référence électronique}

Marjorie Bertin, « Le piège du folklore est totalement dépassé », Horizons/Théâtre [En ligne], 14 | 2019, mis en ligne le 01 juin 2022, consulté le 30 juin 2022. URL : http://journals.openedition.org/ht/1760 : DOI : https://doi.org/10.4000/ht.1760

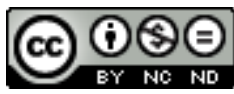

La revue Horizons/Théâtre est mise à disposition selon les termes de la Licence Creative Commons Attribution - Pas d'Utilisation Commerciale - Pas de Modification 4.0 International. 


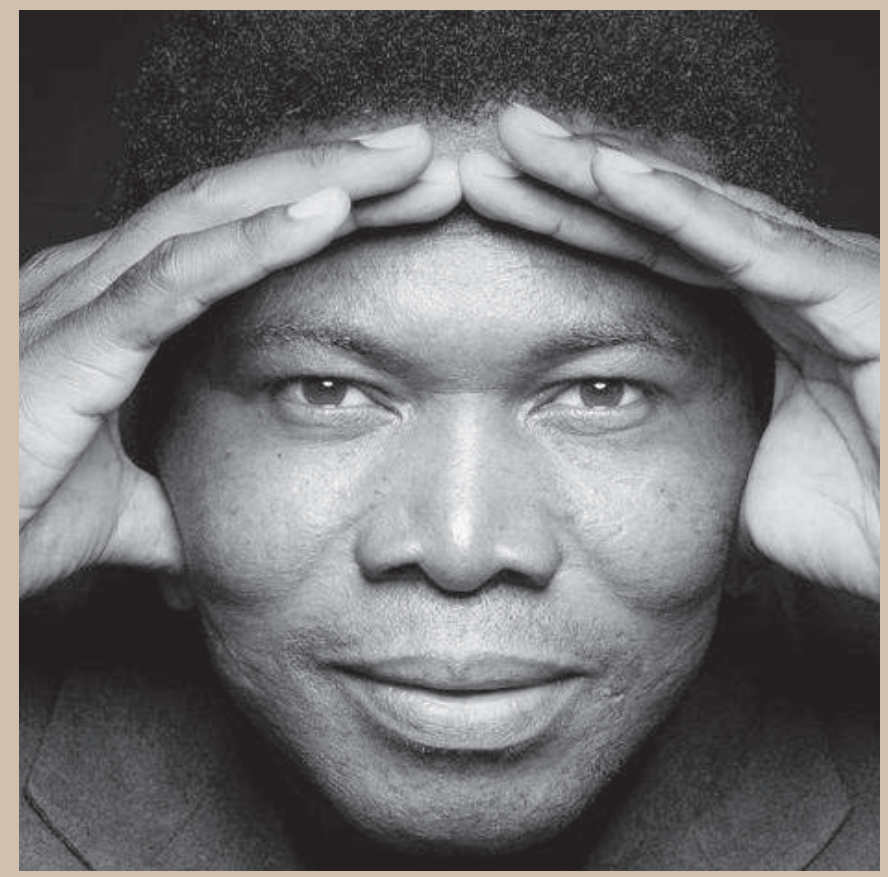

Étienne Minoungou est un comédien, metteur en scène, dramaturge et entrepreneur culturel africain, basé à Ouagadougou. Après des études de sociologie à I'Université de Ouagadougou et quelques années comme enseignant, il choisit de se consacrer au théâtre. Après avoir été directeur artistique du Théâtre de la Fraternité à Ouagadougou, il fonde la compagnie Falinga en 2000. En 2002, il lance à Ouagadougou les Premières résidences d'écriture et de création théâtrales panafricaines : les Récréâtrales. Ce Festival est devenu l'un des espaces les plus importants de la création théâtrale en Afrique.

Ateliers d'écriture, mise en scène, jeux d'acteurs, il propose des résidences à une centaine d'artistes du continent. Ils travaillent sur place, pendant trois à quatre mois, des spectacles qui seront intégrés à la programmation internationale du festival. Ces résidences tentent d'explorer une nouvelle approche de la création dramatique en Afrique, en privilégiant un travail conjoint du texte et de la mise en scène et en inscrivant le tout sur un territoire urbain et dans des cours familiales à Ouagadougou. Comédien, il tourne depuis six ans sur les scènes Européennes et Africaines avec quatre grandes œuvres: M'appelle Mohamed Ali de Dieudonné Niangouna, Cahier d'un retour au pays natal de Césaire, Si nous voulons vivre de Sony Labou Tansi et maintenant Traces, Discours aux nations africaines de Felwine Sarr. II totalise avec ses créations plus de 450 représentations à travers le monde. 


\section{Le piège du folklore est totalement dépassé}

Que pensez-vous de l'appellation « théâtre africain » ou «théâtre subsaharien $\gg$ ?

Il y a un aspect très technique et géographique dans ces termes. Nommer un territoire c'est tout de même plus simple pour le placer sur une carte ! Donc cela ne me gêne pas du tout. Il y avait bien sûr un malaise, à une certaine époque, parce que cela renvoyait à l'idée d'une pratique théâtrale dont les origines seraient importées du théâtre occidental... Cette propension à faire des comparaisons pour mettre une échelle de valeur entre les pratiques sous le Sahara et les pratiques occidentales était gênante car ces comparaisons sous-entendaient aussi des questions comme « est-ce qu'il y a vraiment du théâtre là-bas ? » Aujourd'hui, par la présence des artistes de ces pays sur les plateformes internationales professionnelles, ces débats tendent à être dépassés et définissent plutôt une origine géographique que l'origine des créateurs. Du point de vue des artistes c'est un vieux débat.

Mais tout de même, en parlant de «théâtre africain », on parle d'un continent entier...

Oui, mais c'est très variable, on dit aussi théâtre angolais ou nigérien. Cela dit c'est vrai que certains chercheurs conservent parfois les catégories anthropologiques et ethnologiques qui avaient cours il y a quelques années.

Quel regard portez-vous sur la situation actuelle du théâtre et des dramaturges africains évoluant en Afrique ?

Il y a un foisonnement. Ce « printemps » est sans doute lié à la mise en place des plateformes de création et de recherche théâtrale que l'on retrouve sur tout le Continent. Il y a bien sûr les Récréâtrales, qui est devenue l'une des plateformes les plus importantes du théâtre et a sans doute participé à relancer l'activité théâtrale. Il y a également Univers des mots en Guinée, créé par le dramaturge Hakim Bah et son collègue Bilia Bah. Au Congo, le festival Ça se passe à Kin dirigé par le Tarmac des auteurs, le festival Kitambo à Bamako ; 
le festival Émergences au Niger rassemble lui aussi beaucoup de monde. Toutes ces manifestations permettent aux auteurs de se développer et de diffuser leurs œuvres. Un autre phénomène nouveau, c'est l'édition. De plus en plus d'auteurs africains sont publiés, par les éditions Lansman par exemple, mais aussi par des éditions locales en Afrique. Cela permet aux metteurs en scène de se saisir de textes qui dormaient dans les tiroirs. Il y a aussi des maisons d'édition qui fleurissent un peu partout sur le Continent. L'arrivée du numérique a également permit de faire exister des textes. Ce n'est pas la même chose qu'un travail éditorial classique, mais ces nouvelles plateformes permettent à des auteurs de se publier eux-mêmes. Il y a aussi les maisons d'édition constituées à partir de petits collectifs indépendants qui diffusent les livres dans les librairies. Cela donne une certaine visibilité.

Le fait que RFI ait repris son concours théâtral (le Prix théâtre $\mathrm{RFI}^{1}$ ) en 2014 est aussi une très bonne chose. Ce prix est important car il permet à des auteurs d'être mis en scène et découverts. Au Festival d'Avignon, la création par RFI de « Ça va, ça va le monde $!^{2}$ » offre également un véritable espace de visibilité et de rencontres pour les artistes.

Un autre phénomène que j'observe c'est l'intensification des rencontres entre auteurs et metteurs en scène. Ce ne sont pas forcément des auteurs de théâtre, de plus en plus de textes de romanciers d'Afrique sont portés au théâtre. Ces connivences sont de plus en plus nombreuses et permettent de faire exister des textes qui n'étaient pas destinés à la scène dans un premier temps. Par exemple, Aristide Tarnagda, l'un des plus grands metteurs en scène contemporains en Afrique, travaille en ce moment avec l'auteur congolais Sino Sanza. Cette rencontre produit l'une des déflagrations théâtrales les plus importantes qu'on puisse observer aujourd'hui. Ces nouvelles connivences entre auteurs et metteurs en scène génèrent une nouvelle dynamique. Un des problèmes, il y a quelques années, c'était que les textes venaient du théâtre occidental ; c'est moins le cas aujourd'hui. Il y a de plus en plus d'œuvres du répertoire africain. Et je ne parle même pas des Sony Labou Tansi, Kangni Alem, Dieudonné Niangouna ou Felwin Sarr...

\section{Quels sont les dramaturges africains contemporains sur lesquels vous aimeriez attirer l'attention ?}

Le premier, pour moi, c'est Aristide Tarnagda ; c'est l'un des maîtres en ce moment, à la fois en tant qu'auteur et en tant que metteur en scène. Il y a aussi Sino Sanza, le Béninois Sedjro Giovanni Houansou, qui a été lauréat du prix théâtre RFI en 2018, le Camerounais Edouard Elvis Bvouma, lauréat du Prix 
théâtre RFI en 2017. Ce sont les têtes de proue de la jeune génération. Sans oublier Dieudonné Niangouna bien sûr.

Que représente votre premier passage dans le « In $\gg$ du Festival d'Avignon (transformée en « Semaine d'Art en Avignon » en octobre 2020 en raison de la pandémie de la Covid) avec Traces, Discours aux nations africaines de l'économiste et écrivain sénégalais Felwin Sarr ?

J'étais venu chaque année dans le Off depuis 2015. Même si la Semaine d'art était très différente du Festival d'Avignon du mois de juillet, cela m'a fait très plaisir d'y participer. Cette édition a permis une pleine réception de ce travail et aura certainement des répercussions pour la suite de ce spectacle. C'est aussi une forme de reconnaissance pour un travail amorcé depuis plusieurs années. Cela a été pour moi un moment d'autant plus important que c'est un texte fort qui s'adresse au monde et pas seulement à la jeunesse africaine. C'est un discours qui a besoin d'être entendu aussi dans cette partie du monde avec laquelle nous partageons une longue histoire. Les spectateurs d'Avignon l'ont accueilli avec énormément d'attention et d'émotion. J'en étais très content.

Ce spectacle tournera beaucoup en Europe, ne craignez-vous pas qu'il concerne alors surtout la diaspora, notamment française ?

Non car une longue tournée se prépare sur le Continent. Par ailleurs, je l'ai déjà joué à Ouagadougou, à Dakar et nous serons en tournée au Maroc, au Congo, à Kinshasa donc il fera sa route. Et de toute façon c'est un texte qui va au-delà de son titre et qui s'adresse à tout le monde notamment aux pays avec lesquels l'Afrique a une longue histoire.

Vous travaillez régulièrement en France, vous avez notamment créé la pièce M’appelle Mohamed Ali de Dieudonné Niangouna, au Théâtre Gérard Philipe de Saint-Denis. Saint-Denis est la ville de France dans laquelle il y a le plus de personnes issues de la diaspora africaine. Vous avez également joué ce spectacle dans des collèges et des lycées de la ville. Comment les jeunes vous percevaient-ils ? Comme un acteur africain ? Noir ? Burkinabé ?

Je l'ai joué près de 350 fois dans une vingtaine de pays ! Il y a d'abord la figure de Mohamed Ali qui est emblématique des luttes pour les droits civiques. Le personnage est un héros mondial et les spectateurs s'identifiaient non pas seulement à lui en tant que boxeurs, mais aussi à ses combats politiques. La 
perception des jeunes n'était pas du tout liée à la race ou à la couleur mais plutôt à la force de son combat qui résonne chez eux aujourd'hui. Nous avons aussi beaucoup joué sur la ressemblance physique qui permettait de créer dans l'imaginaire du spectateur une sorte de schizophrénie entre l'acteur et le personnage. Ces deux différentes choses ont sans doute fait que les spectateurs ne se sont pas arrêtés à l'acteur et aux personnages mais aux thématiques très actuelles de la pièce, le désir de liberté, le combat, la résistance, la capacité à dire non. Je crois que « l'acteur qui vient d'Afrique » passait vraiment au second plan. Et puis c'était une performance, un spectacle très physique qui m’a demandé beaucoup d'entraînement. Cela a pu aussi impressionner les jeunes! C'est un spectacle qui est encore joué et programmé aujourd'hui.

Quand on est un artiste africain, est-on obligé d'être reconnu en Europe pour avoir de la visibilité ?

Ce n'est peut-être plus nécessaire parce que beaucoup des artistes que je viens de citer sont déjà connus dans leurs propres pays. Auparavant il fallait « passer par » Paris ou Avignon dans un premier temps pour être reconnu. Maintenant ce n'est plus unilatéral, Avignon aussi vient à Ouagadougou, à Brazzaville, etc. Et pour les artistes c'est vraiment une bonne chose. La labellisation ne passe plus par Paris, Limoges ou Avignon comme autrefois mais c'est un passage qui reste important car cela élargit l'imaginaire des artistes et leur espace de déploiement. Pour moi ça reste important également parce que c'est l'espace reconnu de la francophonie et que cela permet de se déployer aussi dans d'autres pays francophones.

Pour un artiste comme vous, le plus d'un Festival comme Avignon, par rapport à un autre festival international comme Édimbourg, c'est donc la francophonie?

Oui c'est la dimension francophone.

Comment peut-on garder son originalité et sa liberté tout en répandant aux cahiers de charges des producteurs européens ? Surtout qu'il y a une demande spécifique qu'on peut parfois qualifier de folkloriste très prisée en Europe.

Je crois que les producteurs et les programmateurs ont compris que cela ne fonctionne plus aujourd'hui. Les publics voient beaucoup de choses. Le folklore ne marche plus. Ce qui fonctionne c'est l'artiste ou le poète qui a quelque chose à dire, cela devient très important et tous les théâtres et les producteurs 
cherchent une parole singulière. Et elle ne dépend pas d'un habillage qu'on pourrait attendre du Continent. On attend le poète. Je le vois à la manière dont les programmations se font, aux déplacements sur le Contient des programmateurs et des producteurs occidentaux. Ils s'intéressent à la singularité des artistes. Plus personne ne tomberait aujourd'hui dans le piège du folklore, le public sait décoder ce qu'il voit, cela ne marcherait plus de venir en Europe avec des djembés et des danses. C'est totalement dépassé.

\section{Vous avez fondé les magnifiques Récréâtrales de Ouagadagou, à la fois} très prisées par les artistes et la critique internationale tout en étant très populaires. Comment avez-vous réussi à produire cette alchimie ? Est-ce que c'est justement en refusant d'abord d'en faire un « festival » mais plutôt un laboratoire, avec des résidences panafricaines d'écriture, de création et de formation théâtrale en trois sessions tous les deux ans ?

C'est un laboratoire de recherche. Donner du temps aux artistes pour aller jusqu'au bout d'une démarche de création. C'est ça le mot : Résidences d'écriture de création et de recherche théâtrale. Je voulais que ça ne soit pas un festival dans lequel on programme des œuvres déjà faites mais que ça soit un processus qui donne la possibilité aux artistes de travailler sans se soucier de rendez-vous, planning, etc. Et surtout que cela se passe de manière collective. On reste ensemble pendant trois, quatre mois en résidence de travail avec la possibilité de se nourrir mutuellement des démarches des uns et des autres, de renforcer l'imaginaire par des ateliers, de rassembler autour de la table scénographes, metteurs en scène, auteurs.

Mais je voulais aussi que ça ne soit pas un événement déconnecté de la vie sociale et de la respiration de l'environnement dans lequel ces artistes travaillent. Je voulais qu'ils travaillent au cœur même de la ville, parce que le festival, les résidences se déroulent dans les quartiers populaires et quà la fin les représentations se tiennent dans les cours familiales. En amont les gens se fondent avec les artistes. On fait du théâtre pour tous et pour toutes les classes sociales. C'est un lieu de naissance et de déploiement concret, pas théorique. C'est pour ça que ça a pris cette force. Les résidences sont aussi dans les familles, les artistes sont dans la vie familiale, tout en étant à côté d'autres artistes qui font le même travail. Ils se retrouvent, passent de longues semaines avec plusieurs étapes de travail jusqu'aux représentations. Les créations sont en prise directe avec le territoire où sont jouées les représentations. 
Cette démarche s'inscrivait dans le souci de vivifier le théâtre auprès des gens?

C'était de retrouver notre place, parce que c'est vrai qu'il y a vingt ans le théâtre n'appartenait pas au peuple. Il y avait un côté « entre soi ». Or je considère que le théâtre est un espace du débat social et chez nous la communauté est importante. C'est primordial que le théâtre soit dans la rue, les parcs, là où la société se regarde. La rue c'est à la fois un espace intime et ouvert c'est trouver sa place et sa reconnaissance, et puis c'est comme dire à la communauté : on va cuisiner devant vous et on va manger ensemble. Cela fait du bien au public. Au début il y avait une centaine de personnes triées sur le volet aujourd'hui il y a dans les 3000 personnes.

\section{Combien les spectateurs paient-ils pour assister aux spectacles ?}

Il y a une entrée payante, modique, mais elle est là. Il y a un effort de déplacement. C'est 50 francs CFA. Cela représente moins d'un euro.

\section{Comment les artistes sont-ils sélectionnés pour venir aux Récréâtrales ?}

Au début c'était par un appel à candidatures, on recevait des projets et les plus intéressants étaient ensuite sélectionnés par un comité. Aujourd'hui il $y$ a toujours un appel à candidatures, mais cela se prépare aussi beaucoup en amont, c'est parfois des commandes ou de cartes blanches à des artistes qu'on repère. Et puis Aristide Tarnagda a mis en place le programme ELAN qui recrute chaque année une trentaine de jeunes artistes (metteurs en scène, auteurs, scénographes, comédiens et techniciens) qui suivent le même parcours de formation pendant un an. Cela fonctionne comme une bourse, ils sont entièrement pris en charge, transport, hébergement, perdiem, etc. Les œuvres les plus abouties reviennent ensuite pour être créées aux Récréthéâtrales. L'objectif est de fournir un espace temps de formation et de recherche à des artistes professionnels tout en favorisant les échanges et les rencontres.

Le théâtre que vous jouez et défendez est très physique, très visuel... Est-ce que cela peut résister face à la vague des nouvelles technologies sur les plateaux ? Aux acteurs hologrammes à la réalité virtuelle, aux écrans ?

Oui, au contraire! Je pense que le théâtre reprend sa place en ce moment, sa place étant de rassembler. Rien ne remplace le fait d'être ensemble. Les technologies particularisent chacun dans sa petite zone et engendrent un risque communautaire. La force du théâtre, c'est de réussir à réunir dans un même espace plusieurs personnes d'origines diverses qui regardent la même 
chose, qui vivent une expérience, certes particulière, mais ensemble. C'est ce qui est formidable dans les Récréâtrales, les spectacles se font dans les cours des gens. C'est dans les cours des maisons dans lesquels ils vivent que sont rassemblés des gradins et 200 personnes. C'est tellement fort que ça génère d'autres publics, le jeu redessine la place du théâtre au sein de la communauté et donne une nouvelle dynamique. Les artistes sont attendus, reconnus. Avec l'arrivée d'internet et de Canal +, les rassemblements s'amenuisaient. Aujourd'hui on redécouvre le plaisir de se retrouver tous ensemble.

\section{Par Marjorie Bertin}

\section{Notes}

1. Le « Prix Théâtre RFI » a pour objectif de promouvoir la richesse des écritures dramatiques contemporaines francophones du Sud et de favoriser le développement de carrière de jeunes auteurs, écrivant en français. RFI et ses partenaires offrent au lauréat un soutien professionnel et une exposition médiatique à travers une dotation financière attribuée par la SACD ; l’organisation d'une résidence en France, en 2015, à la Maison des Auteurs de Limoges et au Théâtre de l'Aquarium, financée par l'Institut français et accompagnée par l'association Beaumarchais-SACD ; une promotion du texte et une mise en ondes sur les antennes de RFI.

2. Depuis 2012, le cycle de lectures « Ça va, ça va le monde ! » créé et présenté par Pascal Paradou fait découvrir des auteurs émergents sur la scène internationale. Les pièces sélectionnées sont diffusées sur les ondes de RFI, tous les samedis du 25 juillet au 29 août. 